\title{
DEPOLARIZATION-INDUCED RELEASE OF ADENOSINE 5'-TRIPHOSPHATE FROM ISOLATED VARICOSITIES DERIVED FROM THE MYENTERIC PLEXUS OF THE GUINEA PIG SMALL INTESTINE ${ }^{1}$
}

\author{
THOMAS D. WHITE ${ }^{2}$ AND RONALD A. LESLIE \\ Departments of Pharmacology and Anatomy, Dalhousie University, Halifax, Nova Scotia, Canada B3H $4 H 7$
}

Received June 18, 1981; Revised October 6, 1981; Accepted October 8, 1981

\begin{abstract}
In order to study the release of ATP from a neuronal preparation thought to be "purinergic," isolated varicosities were prepared from myenteric plexus by mincing and homogenizing the longitudinal muscle of guinea pig ileum in $0.32 \mathrm{M}$ sucrose. The presence of varicosities within the crude preparation $\left(\mathrm{P}_{2}\right)$, isolated by differential centrifugation, was confirmed by electron microscopy and by the presence of occluded lactate dehydrogenase as a cytoplasmic marker. Varicosities were purified further from the $\mathrm{P}_{2}$ fraction on a discontinuous sucrose density gradient and characterized morphologically. Varicosities resembling cholinergic, "purinergic," and adrenergic axonal terminals were identified. The release of ATP from both crude and purified varicosities was detected by monitoring the light produced when the released ATP reacted with firefly luciferin-luciferase which was present in the incubation medium. Elevated extracellular $\mathrm{K}^{+}$or $\mathrm{Rb}^{+}$caused the release of ATP, whereas elevated $\mathrm{Na}^{+}$and $\mathrm{Li}^{+}$did not. The release of ATP by either elevated $\mathrm{K}^{+}$or by the depolarizing drug, veratridine, was $\mathrm{Ca}^{2+}$ dependent. This requirement of extracellular $\mathrm{Ca}^{2+}$ for veratridine-induced release of ATP from isolated myenteric varicosities contrasted strikingly with that for brain synaptosomes, where the release of ATP induced by veratridine was increased rather than decreased in a $\mathrm{Ca}^{2+}$-free medium. The characteristics of ATP release from myenteric varicosities are consistent with a possible neurotransmitter function for ATP in myenteric plexus, although it is also possible that ATP is co-released with some other neurotransmitter.
\end{abstract}

It has been suggested that a purine nucleotide, possibly ATP, might be the non-adrenergic, non-cholinergic inhibitory neurotransmitter in vertebrate gastrointestinal smooth muscle (see Burnstock, 1979, for review). Exogenously applied ATP causes mechanical responses which resemble those produced by electrical field stimulation of these preparations (Burnstock et al., 1970). Moreover, tetrodotoxin-sensitive release of radiolabeled adenosine derivatives has been demonstrated during field stimulation of these tissues following incubation with $\left[{ }^{3} \mathrm{H}\right]$ adenosine (Su et al., 1971; Rutherford and Burnstock, 1978). A small release of endogenous ATP also has been reported in superfusates from electrically stimulated Taenia coli (Burnstock et al., 1978). It is likely that much of the ATP is very rapidly degraded extracellularly following its release (Satchell and Burnstock, 1971).

\footnotetext{
'This work was supported by the Medical Research Council of Canada. We thank Diana Webb and Glenn Smith for their expert technical assistance.

${ }^{2}$ To whom correspondence should be addressed at Department of Pharmacology, Dalhousie University, Halifax, Nova Scotia, Canada B3H $4 \mathbf{H} 7$.
}

We have developed a technique which allows us to detect directly and continuously the release of ATP from rat brain synaptosomal preparations by monitoring the light produced when the released ATP reacts with firefly luciferin-luciferase present in the incubation medium (White, 1977, 1978; White et al., 1980; Potter and White, 1980). This technique offers a number of advantages over other methods in that it is sensitive and specific for ATP, it permits continuous short term temporal observations of release, and it should detect ATP before the nucleotide can be degraded by ecto-ATPases.

Recently, the preparation of isolated varicosities from the myenteric plexus of guinea pig ileum has been reported by several laboratories (Jonakait et al., 1979; Dowe et al., 1980; Briggs and Cooper, 1981). The use of isolated varicosities to study the characteristics of ATP release from myenteric neurons should offer an advantage over other preparations in that release from possible non-neuronal sites, such as muscle, would be excluded. In the present study, we developed a simple method for preparing and isolating varicosities from the myenteric plexus of guinea pig ileum, assessed the purity of the preparations, and characterized the varicosities morpho- 
logically using electron microscopy. We then studied the depolarization-induced release of ATP from these preparations. Portions of this work have been reported in preliminary forms previously (White and Webb, 1980a, b).

\section{Materials and Methods}

Drugs and chemicals. $\beta$-Nicotinamide-adenine dinucleotide $(\beta-\mathrm{NADH})$, sodium pyruvate, $\mathrm{D}$-luciferin, crude firefly extract (FI.F-50), and disodium ATP were purchased from Sigma Chemical Co., St. Louis. Veratridine was supplied by Aldrich Chemical Co., Milwaukee and tetrodotoxin was from Calbiochem, San Diego. Aqueous glutaraldehyde $(25 \%)$, paraformaldehyde, sodium cacodylate trihydrate, $\mathrm{OsO}_{4}$, and Spurr epoxy resin for electron microscopy were purchased from TAAB Laboratories, Reading, England. Uranyl acetate was supplied by Fisher Scientific Co., Montreal.

Preparation of myenteric varicosities. Myenteric varicosities were prepared from guinea pigs (High Oak Ranch, Goodwood, Ontario) of either sex weighing 300 to $400 \mathrm{gm}$ by a modification of the method of Jonakail et al. (1979). In their study, they minced and homogenized the longitudinal muscle from guinea pig ileum in $0.3 \mathrm{M}$ sucrose buffered to $\mathrm{pH} 7.4$ with $0.1 \mathrm{M}$ sodium phosphate buffer containing $1 \mathrm{~mm}$ EGTA. In preliminary experiments, we greatly improved the yield of isolated varicosities, as assessed morphologically by electron microscopy and by the presence of occluded lactate dehydrogenase as a cytoplasmic marker, by mincing and homogenizing the tissue in $0.32 \mathrm{M}$ sucrose which did not contain sodium phosphate buffer and EGTA.

Longitudinal muscle with attached myenteric plexus was removed from the ileum of each of three guinea pigs (Paton and Zar, 1968) into $0.32 \mathrm{M}$ sucrose, blotted, and then minced thoroughly with scissors before being homogenized in $10 \mathrm{ml}$ of $0.32 \mathrm{M}$ sucrose with seven strokes of a Teflon-glass homogenizer (clearance, $0.25 \mathrm{~mm}$ ). The homogenates from the three guinea pigs were pooled and centrifuged at $1,000 \times g$ for $10 \mathrm{~min}$, and the supernatant was decanted and saved. The remaining pellet was resuspended in $0.32 \mathrm{M}$ sucrose and centrifuged at $1,000 \times \mathrm{g}$ for $10 \mathrm{~min}$. The resultant supernatant was pooled with the original supernatant and centrifuged at $20,000 \times g$ for 30 $\mathrm{min}$, and the pellet $\left(\mathrm{P}_{2}\right)$ was suspended finally in $8 \mathrm{ml}$ of Krebs-Henseleit medium containing $111 \mathrm{~mm} \mathrm{NaCl}, 26.2$ $\mathrm{mm} \mathrm{NaHCO}, 1.2 \mathrm{mM} \mathrm{NaH}, \mathrm{PO}_{4}, 4.7 \mathrm{~mm} \mathrm{KCl}, 1.8 \mathrm{~mm}$ $\mathrm{CaCl}_{2}, 1.2 \mathrm{mM} \mathrm{MgCl} 2,11 \mathrm{~mm}$ glucose and gassed with $95 \%$ $\mathrm{O}_{2}, 5 \% \mathrm{CO}_{2}$ to maintain a $\mathrm{pH}$ of 7.4. In some cases, the pellet was resuspended in a $\mathrm{Ca}^{2+}$-free medium otherwise identical to the above. The suspension was preincubated for $30 \mathrm{~min}$ at $37^{\circ} \mathrm{C}$ with periodic gassing before studies of the release of ATP were performed.

Purification of myenteric varicosities on sucrose density gradients. The crude material containing isolated varicosities $\left(\mathrm{P}_{2}\right)$ from four guinea pigs was resuspended in $12 \mathrm{ml}$ of $0.32 \mathrm{M}$ sucrose and $2 \mathrm{ml}$ were layered onto each of six density gradients consisting of $5 \mathrm{ml}$ of $0.8 \mathrm{M}$ sucrose on $5 \mathrm{ml}$ of $1.2 \mathrm{M}$ sucrose. The gradients were centrifuged at $150,000 \times g$ for $60 \mathrm{~min}$ in an International B60 centrifuge (rotor SB 283) and the material at the $0.8: 1.2 \mathrm{M}$ sucrose interface was removed in $3 \mathrm{ml}$ using a syringe and needle with a curved tip. The pooled mate- rial, corresponding to purified varicosities, was diluted to $40 \mathrm{ml}$ with $0.32 \mathrm{M}$ sucrose and centrifuged at $20,000 \times \mathrm{g}$ for $30 \mathrm{~min}$. The pellets at the bottom of the $1.2 \mathrm{M}$ sucrose also were resuspended with $40 \mathrm{ml}$ of $0.32 \mathrm{M}$ sucrose and centrituged at $20,000 \times g$ for 30 min to yield a mitochondrial fraction. The material containing varicosities was resuspended in $2 \mathrm{ml}$ of incubation medium (about $0.5 \mathrm{mg}$ of protein $/ \mathrm{ml}$ ) and the mitochondrial material was resuspended in $6 \mathrm{ml}$ of medium (about $0.5 \mathrm{mg}$ of protein $/ \mathrm{ml}$ ); both preparations were preincubated for $30 \mathrm{~min}$ before being used in studies of the release of ATP.

Preparation of synaptosomes from brain. Synaptosomes were prepared from the whole brains of two guinea pigs. Each brain was homogenized in $26 \mathrm{ml}$ of $0.32 \mathrm{M}$ sucrose by 15 strokes of a loose-fitting Teflon-glass homogenizer revolving at $800 \mathrm{rpm}$. The homogenate was divided into equal portions and centrifuged at $1,000 \times g$ for $10 \mathrm{~min}$, and the resulting supernatants were recentrifuged at $12,000 \times g$ for 20 min to yield the crude synaptosomal pellets. These were resuspended with a total of $80 \mathrm{ml}$ of incubation medium and preincubated for $30 \mathrm{~min}$ before being used to study the release of ATP.

Detection of ATP release. The release of ATP was monitored directly and continuously as described previously (White, 1978). Briefly, $0.5 \mathrm{ml}$ of the appropriate suspension was placed into a $6 \times 50 \mathrm{~mm}$ cylindrical cuvette with $0.02 \mathrm{ml}$ of firefly luciferin-luciferase prepared by mixing equal volumes of synthetic D-luciferin $\left(5 \mathrm{mg} / \mathrm{ml}\right.$ of $\mathrm{H}_{2} \mathrm{O}$ ) with crude firefly extract $(25 \mathrm{mg} / \mathrm{ml}$ of $\mathrm{H}_{2} \mathrm{O}$; Sigma FLE 50). The myenteric varicosities or brain synaptosomes were depolarized by rapidly injecting 10 $\mu \mathrm{l}$ of either $125 \mathrm{mM} \mathrm{KCl}$ or $2.7 \mathrm{~mm}$ veratridine (increasing the final concentration of $\mathrm{KCl}$ by $23 \mathrm{~mm}$ or that of veratridine to $50 \mu \mathrm{M})$. The release of ATP was detected by monitoring the light produced by the ATP-luciferinluciferase reaction in an Aminco Chemglow chemiluminometer. Standard solutions of ATP in incubation medium also were injected to establish the sensitivity of detection of ATP in the system.

Electron microscopy. Material containing isolated myenteric varicosities was incubated for $30 \mathrm{~min}$ in KrebsHenseleit medium and then centrifuged to a pellet and resuspended in a fixative solution for electron microscopy. The fixative consisted of $1 \%$ paraformaldehyde and $1 \%$ glutaraldehyde in $0.1 \mathrm{M}$ sodium cacodylate buffer at $\mathrm{pH}$ 7.3. After fixing at room temperature for $1 \mathrm{hr}$, the varicosities were centrifuged to a pellet and left in the fixative for a further $20 \mathrm{hr}$. The pellet then was washed twice in $0.2 \mathrm{M}$ cacodylate buffer for a further $2 \mathrm{hr}$. Following another wash in $0.2 \mathrm{~m}$ buffer, the preparation was dehydrated in an ascending ethanol series and propylene oxide and embedded in Spurr epoxy resin. Some samples were given a tertiary fixation in $1 \%$ uranyl acetate in $70 \%$ ethanol before dehydration and embedding. The embedded pellets were thin-sectioned with a diamond knife and sections were stained with lead citrate (Reynolds, 1963) or saturated aqueous uranyl acetate followed by lead citrate. Sections were examined in a Zeiss EM10A electron microscope.

Determination of occluded lactate dehydrogenase (EC 1.1.1.27; $L D H$ ). Occluded LDH was determined on the purified varicosities and mitochondrial material as a marker of cytoplasm enclosed within a cell membrane 

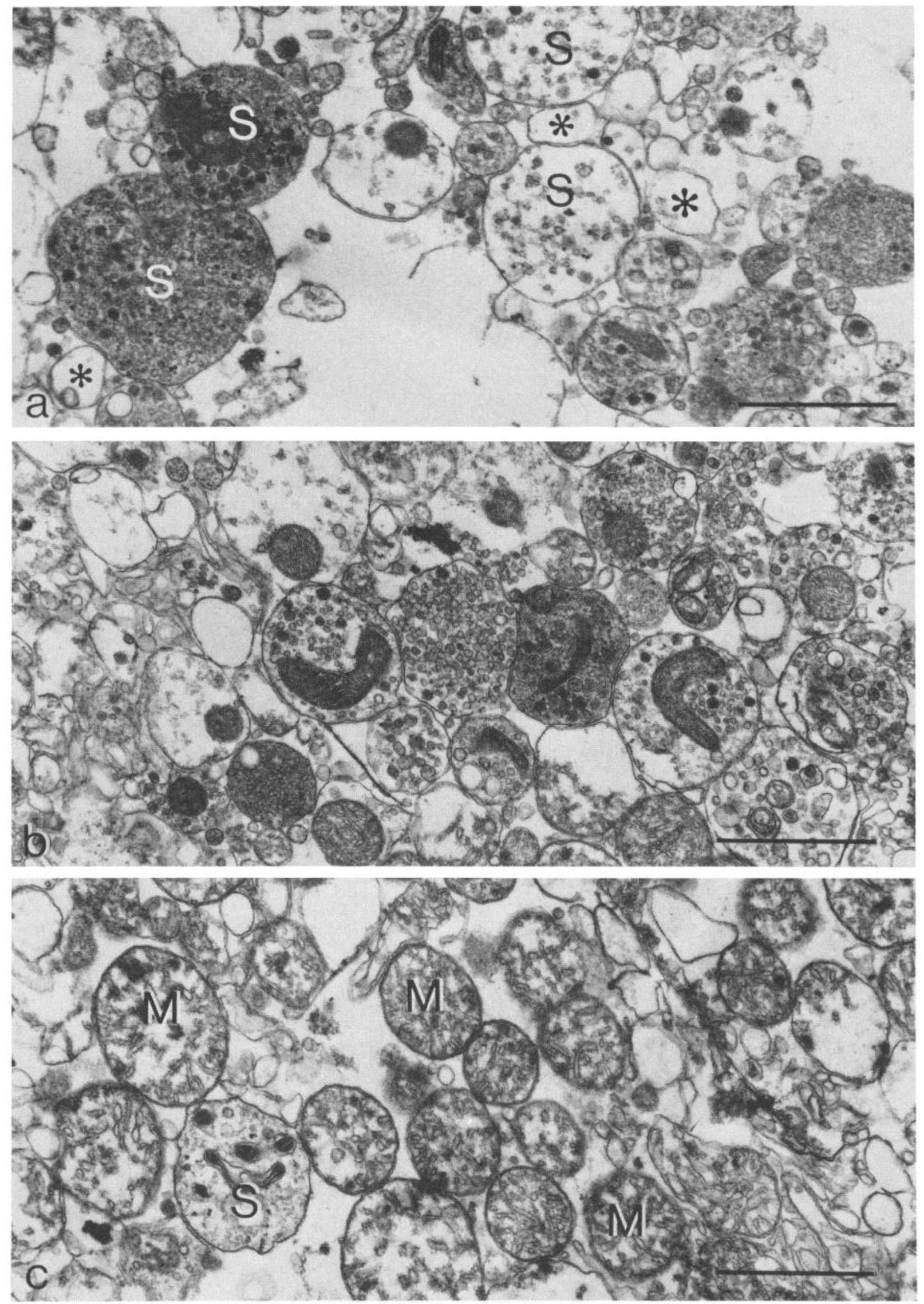

Figure 1. $a$, Low power electron micrograph of the crude myenteric varicosity fraction $\left(\mathrm{P}_{2}\right)$. A number of varicosities $(S)$ of various types are visible as well as membranous profiles with electron-lucent centers (*) and other cellular debris. Bar $=1 \mu \mathrm{m}$. $b$, Purified varicosities fraction. This fraction is enriched with varicosities of various types. Free mitochondria are rare. Bar $=1 \mu \mathrm{m}$. $c$, Mitochondrial fraction. Although a few varicosities $(S)$ are present in this fraction, most of the profiles are free mitochondria $(M) . B a r=1 \mu \mathrm{m}$. 
(Wroblewski and LaDue, 1955; Whittaker and Barker, 1972). One hundred microliters of suspension were added to $2.8 \mathrm{ml}$ of $100 \mathrm{~mm}$ potassium phosphate buffer $(\mathrm{pH} 7.5)$ containing $50 \mathrm{~mm} \mathrm{NaCl}$ and $0.1 \mathrm{~mm} \mathrm{NADH}$ followed by $100 \mu \mathrm{l}$ of $22.7 \mathrm{~mm}$ sodium pyruvate, and the rate of fall in optical density at $340 \mathrm{~nm}$ was measured in a Unicam SP800 spectrophotometer at $22^{\circ} \mathrm{C}$ against a water blank. One LDH unit was defined as the change in optical density per min $\times 1000$. LDH activity was determined on preparations before and after disruption by adding 10 $\mu \mathrm{l}$ of $10 \%$ Triton X-100 to the cuvette with mixing. 'The difference between the two values for LDH represented occluded LDH or the amount of LDH contained within the cytoplasm. The occluded LDH activity was expressed finally as units of LDH activity per mg of protein.

Protein. Protein was determined by the method of Hartree (1972).

\section{Results}

Preparation, isolation, and characterization of myenteric varicosities. When examined in the electron microscope, the crude preparation of varicosities $\left(\mathrm{P}_{2}\right)$ was seen to contain many nerve terminals with intact, resealed plasma membranes (Fig. 1a). These varicosities ranged in size from about 0.7 to $1.5 \mu \mathrm{m}$ in diameter and contained various types of vesicles, small mitochondria, and sometimes glycogen granules (Fig. 2, $a$ to $f$ ). Other components visible in the preparation were free mitochondria, a few free vesicles similar to those seen in terminals, large (about $1 \mu \mathrm{m}$ in diameter) membranous profiles with electron-lucent centers, and membranous debris. Some of the membranes had resealed to form small $(40 \mathrm{~nm}$ to $0.5 \mu \mathrm{m}$ ), round or pleomorphic profiles (Fig. 1a) similar to the microsomes reported by Dowe et al. (1980).

The purified myenteric varicosities isolated at the 0.8:1.2 $\mathrm{M}$ interface on sucrose density gradients contained large proportions of intact terminals, with few other components contaminating the preparation (Fig. $1 b$ ). These preparations facilitated the study of terminal types as the latter were numerous and very well preserved. The material which sedimented to the bottom of the 1.2 M sucrose contained primarily free mitochondria whose average diameter was about $0.75 \mu \mathrm{m}$ (Fig. 1c). Some varicosities were visible in this fraction, but they were infrequent. Analysis of the occluded lactate dehydrogenase contents of the purified varicosities and mitochondrial fractions, as a measure of the amount of cytoplasm

\section{TABLE I}

Occluded lactate dehydrogenase content of subcellular fractions

Purified myenteric varicosities and mitochondria were prepared and isolated, and their occluded lactate dehydrogenase contents were determined as described under "Materials and Methods." One LDH unit $=$ change in optical density $/ \mathrm{min} \times 1000$. Each value is the mean \pm SEM of four experiments.

\begin{tabular}{cc}
\hline \multicolumn{1}{c}{ Fraction } & $\begin{array}{c}\text { Occluded } \mathrm{LDH} / \mathrm{mg} \\
\text { of Protein }\end{array}$ \\
\hline $\begin{array}{c}\text { Purified varicosities } \\
(0.8: 1.2 \mathrm{M} \text { interface of sucrose gradient) }\end{array}$ & $420 \pm 31$ \\
$\begin{array}{c}\text { Mitochondrial fraction } \\
\text { (pellet below } 1.2 \mathrm{M} \text { sucrose) }\end{array}$ & $171 \pm 34$ \\
\hline
\end{tabular}

enclosed by cell membranes (Johnson and Whittaker, 1963), confirmed that the purified varicosity preparation was enriched in nerve terminals (Table I).

The most frequently observed type of terminal seen in both crude $\left(\mathrm{P}_{2}\right)$ and purified varicosity preparations contained mainly small, round vesicles 40 to $60 \mathrm{~nm}$ in diameter with fairly electron-lucent centers. Often several larger vesicles, 75 to $100 \mathrm{~mm}$ in dianeler, were present within these same terminals, and these contained an electron-dense core sometimes surrounded by a clear halo (Fig. 2a). The vesicles were quite loosely packed within this type of terminal. Often, one or two small mitochondria were visible within these terminals.

Several other types of terminal were seen with less frequency than those described above. These varied a great deal in the density of packing of vesicles and in the electron opacity of the cytoplasm as well as in the proportion of small, clear to large, dense core vesicles contained within them. Some contained numerous large, dense core vesicles (Fig. 2b). Others contained large dense core vesicles and clusters of glycogen granules (Fig. $2 c$ ). Neurotubules were observed sometimes (Fig. 2c). A less common type of terminal contained a large number of tightly packed small, clear vesicles in an electron-dense cytoplasm (Fig. 2d).

Very rarely, terminals were seen which contained an unusually large number of small mitochondria surrounded by a few small, clear vesicles, very few large dense core vesicles, and clusters of glycogen granules (Fig. $2 e$ ). Other rare types of terminals seen were those containing a few clear, flattened vesicles found in company with mainly small, round, clear vesicles. The flattened vesicles had dimensions of approximately $20 \times 50$ nm (Fig. 2f).

Release of ATP during depolarization of crude preparations of myenteric varicosities. Depolarization of crude myenteric varicosities $\left(\mathrm{P}_{2}\right)$ with either veratridine or elevated extracellular $\mathrm{K}^{+}$caused the release of ATP (Fig. 3). Release by veratridine was blocked by the prior administration of the specific $\mathrm{Na}^{+}$channel blocker, tetrodotoxin (Narahashi et al., 1964), indicating, in this case, that the release was mediated by the opening of $\mathrm{Na}^{+}$ channels (Fig. 3). In contrast, the release of ATP by elevated $\mathrm{K}^{+}$was unaffected by tetrodotoxin (Fig. 3), indicating that it was not mediated by $\mathrm{Na}^{+}$channels. This release of ATP by elevated $\mathrm{K}^{+}$was not due to the increased tonicity of the medium since equivalent concentrations of $\mathrm{LiCl}$ and $\mathrm{NaCl}$ did not release ATP (Fig. 4). However, elevating the extracellular $\mathrm{RbCl}$ concentration caused a release of ATP which was identical to that observed with elevated $\mathrm{K}^{+}$. Elevated extracellular $\mathrm{Rb}^{+}$, like $\mathrm{K}^{+}$, has been shown previously to depolarize nerve (Feng and Liu, 1949).

Release of ATP from purified myenteric varicosities. The release of ATP was found to occur primarily from the fraction enriched with nerve terminals (Fig. 5a) as assessed morphologically (Fig. $1 b$ ) and containing most of the occluded lactate dehydrogenase activity (Table I). The purified mitochondrial fraction (Fig. 1c) released very little ATP when exposed to either elevated extracellular $\mathrm{K}^{+}$or veratridine (Fig. $5 b$ ). These findings justify the use of the crude $\mathrm{P}_{2}$ material for studying the release of ATP from myenteric varicosities. 

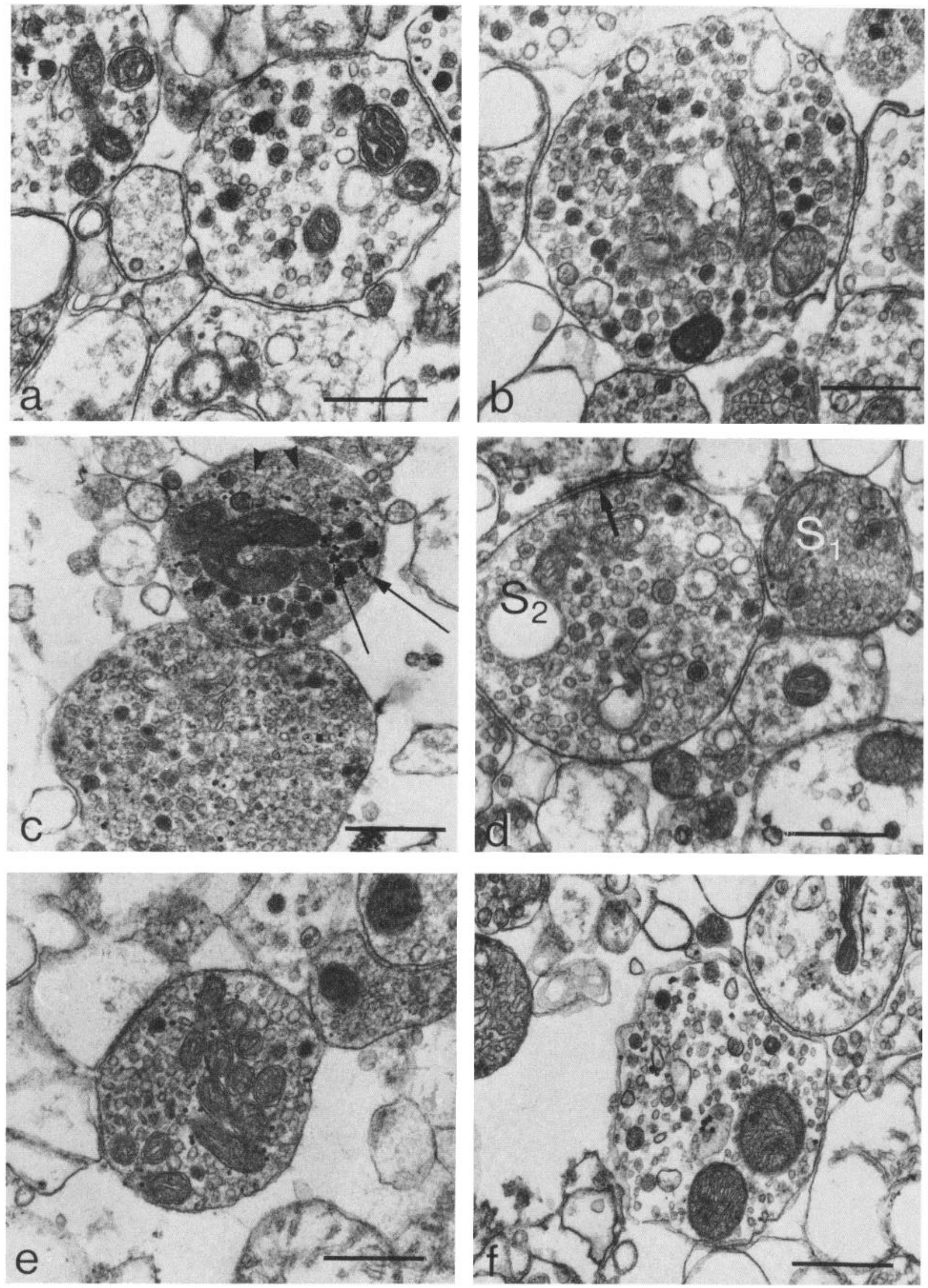

Figure 2. $a$, Shown is an example of the most common type of nerve terminal ("type 1" of Furness and Costa, 1980) which contains small, round vesicles with relatively electron-lucent cores and some larger dense core vesicles which often have an 


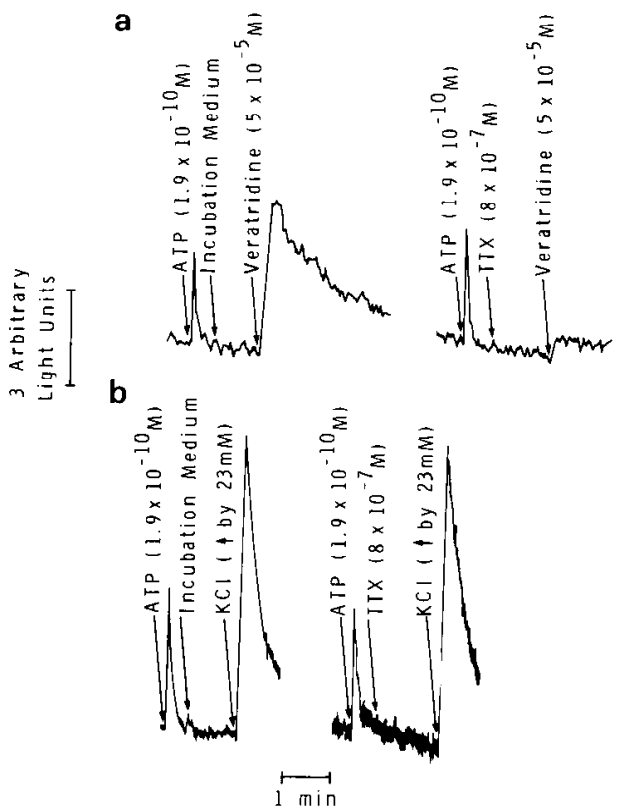

Figure 3. Release of ATP from a crude preparation of myenteric varicosities. Crude varicosities $\left(P_{2}\right)$ were prepared as described under "Materials and Methods." a, A stock solution of $27 \mathrm{~mm}$ veratridine in ethanol was diluted $1: 10$ with incubation medium and $10 \mu \mathrm{l}$ of the latter solution were injected rapidly into the suspension, giving a final concentration of $5 \times 10^{-5} \mathrm{M}$ veratridine. Control injections of equivalent concentrations of ethanol did not produce chemiluminescence. Tetrodotoxin (TTX) in incubation medium was injected to give a final concentration of $8 \times 10^{-7} \mathrm{M}$. A stock ATP solution in incubation medium was injected to give a final ATP concentration of 1.9 $\times 10^{-10} \mathrm{M}$ in the cuvette. The results shown are typical of three experiments. $b$, In other experiments, extracellular $\mathrm{KCl}$ was increased by $23 \mathrm{~mm}$. Tetrodotoxin had no effect on $\mathrm{K}^{+}$-induced release of ATP. The results shown were observed in three experiments.

$\mathrm{Ca}^{2+}$ dependence for depolarization-induced release of ATP from myenteric varicosities. The depolarizationinduced release of a number of putative neurotransmitters from brain synaptosomes has been shown to be $\mathrm{Ca}^{2+}$ dependent (Blaustein et al., 1972; DeBelleroche and Bradford, 1972). Moreover, the $\mathrm{K}^{+}$-induced release of ATP from synaptosomes prepared from rat brain has been shown to be $\mathrm{Ca}^{2+}$ dependent (White, 1978).

The release of ATP by elevated $K^{\prime}$ was diminished

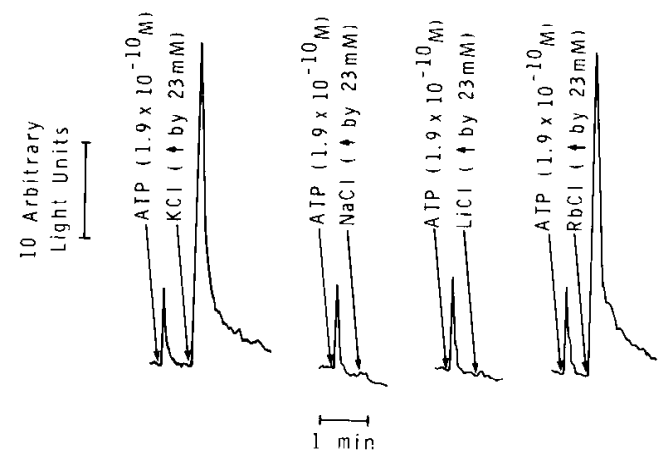

Figure 4. Specificity of release of ATP by monovalent cations. Crude varicosities $\left(\mathrm{P}_{2}\right)$ were prepared as described under "Materials and Methods." Extracellular $\mathrm{KCl}, \mathrm{NaCl}, \mathrm{LiCl}$, or $\mathrm{RbCl}$ were increased by $23 \mathrm{~mm}$ by rapidly injecting $10 \mu \mathrm{l}$ of their stock solutions. A stock solution of ATP in incubation medium was injected to give a final concentration of $1.9 \times 10^{-10}$ M. The results shown were observed in five experiments.

when myenteric varicosities were resuspended and preincubated for 30 min in a medium lacking $\mathrm{CaCl}_{2}$ (Fig. 6a). The addition of $1 \mathrm{~mm}$ EGTA to chelate residual extracellular $\mathrm{Ca}^{2+}$ completely abolished $\mathrm{K}^{+}$-induced release of ATP. The restoration of extracellular $\mathrm{CaCl}_{2}$ to varicosilies preincubated in a $\mathrm{Ca}^{2+}$-free medium restored the $\mathrm{K}^{+}$induced release of ATP to levels even higher than the control values, indicating that preincubation in $\mathrm{Ca}^{2+}$-free medium had not damaged the varicosities irreversibly.

The release of ATP by veratridine also was greatly diminished from myenteric varicosities incubated in a medium lacking $\mathrm{CaCl}_{2}$ (Fig. $6 b$ ). The addition of $1 \mathrm{~mm}$ EGTA to chelate residual $\mathrm{Ca}^{2+}$ completely abolished veratridine-induced release. The restoration of extracellular $\mathrm{CaCl}_{2}$ to varicosities preincubated in a $\mathrm{Ca}^{2+}$-free medium restored veratridine-induced release of ATP to control levels.

This requirement of extracellular $\mathrm{Ca}^{2+}$ for veratridineinduced release of ATP from myenteric varicosities contrasts strikingly with that observed previously for rat brain synaptosomes, where veratridine-induced release of ATP was increased rather than decreased in the absence of extracellular $\mathrm{Ca}^{2+}$ (White, 1978). In order to determine if this discrepancy was due to the different preparations of isolated nerve terminals or to the different species studied, veratridine-induced release of ATP was determined from crude synaptosomal preparations

electron-lucent halo around the core. Such profiles usually contain a few small mitochondria. $B a r=0.5 \mu \mathrm{m}$. $b$, Some varicosities contained a large number of large dense core vesicles ("type 4" of Furness and Costa, 1980). Most, but not all, of these vesicles had an electron-lucent halo around the dense core. A few small, clear vesicles or small vesicles with only slightly electron-dense cores were usually visible in these profiles. $B a r=0.5 \mu \mathrm{m}$. $c$, Some varicosities contain a large number of glycogen granules (arrows) and may correspond to "type 7" of Furness and Costa (1980). Both varicosities visible here contain a large number of dense core vesicles. Note the neurotubules (arrowheads) that are easily recognizable in one of the profiles. Bar $=0.5 \mu$ m. $d$, Another population of varicosities contained a large number of small, clear vesicles compacted together in a relatively electron-dense axoplasmic matrix $\left(S_{1}\right)$. This type could correspond to "type 8" of Furness and Costa (1980). A varicosity $\left(S_{2}\right)$ with an obvious synaptic region (arrow) is also visible in this field. Such synaptic regions were rare in varicosity preparations of myenteric plexus. $B a r=0.5 \mu \mathrm{m}$. $e$, Very rarely, varicosities were encountered which contained large numbers of small mitochondria. This type corresponds to "type 6" of Furness and Costa (1980). Bar $=0.5 \mu \mathrm{m}$. $f$, Occasionally, varicosities were seen with small, clear, flattened or pleomorphic vesicles interspersed with a few large dense core vesicles. These correspond to "type 3 " terminals of Furness and Costa (1980). Bar $=0.5 \mu \mathrm{m}$. 


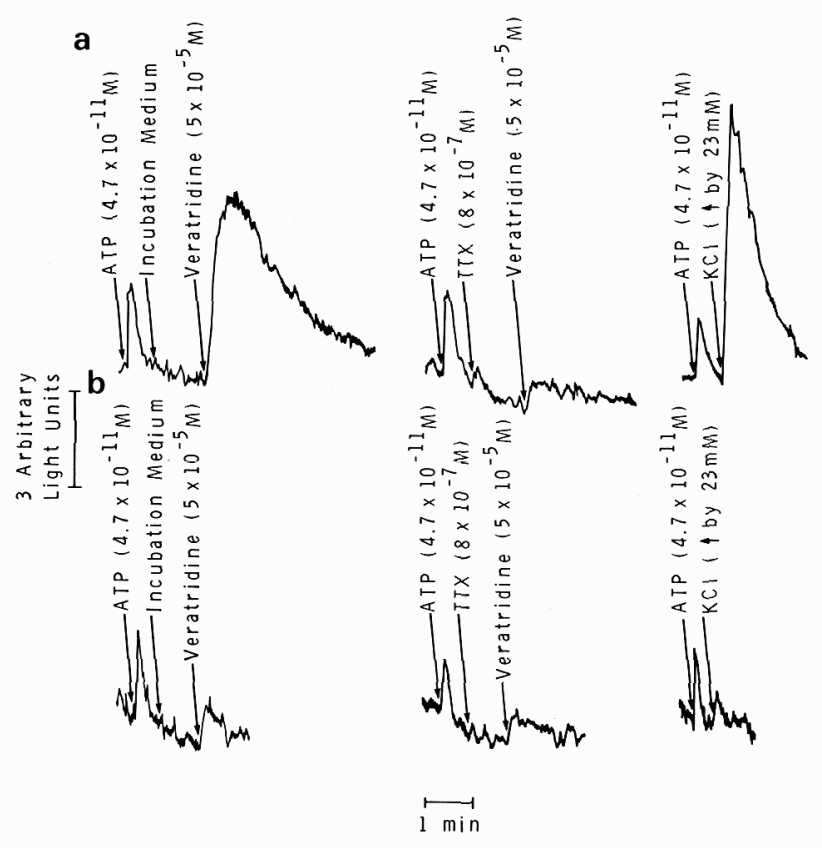

Figure 5. Release of ATP from purified varicosities and mitochondrial preparations. Myenteric varicosities were prepared and purified on a discontinuous sucrose density gradient as described under "Materials and Methods." The material at the 0.8:1.2 M sucrose interface was shown morphologically to contain numerous varicosities (Fig. 1b) and was enriched in occluded lactate dehydrogenase (Table I). The pellet of material of the bottom of the $1.8 \mathrm{M}$ sucrose contained mostly mitochondria (Fig. 1c). $a$, Veratridine and elevated extracellular $\mathrm{K}^{+}$ released ATP from the purified myenteric varicosity preparation. $b$, Very little release of ATP was observed when the corresponding mitochondrial fraction was depolarized with veratridine or elevated extracellular $\mathrm{K}^{+}$. Detection of ATP injected into the cuvette was similar for both preparations. The results shown were observed in seven experiments. TTX, tetrodotoxin.

from the myenteric plexuses and brains of guinea pigs. As with rat brain synaptosomes (White, 1978), veratridine-induced release of ATP from guinea pig brain synaptosomes was actually augmented, rather than reduced, in a $\mathrm{Ca}^{2+}$-free medium (Fig. 7). The restoration of $\mathrm{CaCl}_{2}$ to the incubation medium restored veratridine-induced release to its control level. In contrast, the veratridineinduced release of ATP from myenteric varicosities prepared from the same guinea pigs was greatly reduced in a $\mathrm{Ca}^{2+}$-free medium (Fig. 7).

\section{Discussion}

Characterization of the myenteric varicosity preparations. Most of the types of terminals described by Furness and Costa (1980) in their review of the neuron types found in the enteric nervous system were observed in the preparations of isolated myenteric varicosities used in the present study. The most common type of terminal seen in our preparations of varicosities was also the most common type seen in sections of intact myenteric plexus-namely, "type 1" terminals containing mainly small, round, clear core vesicles (Furness and Costa, 1980). These terminals are thought to be cholinergic (Furness and Costa, 1980).

“Type 2" terminals containing small, round, dense core vesicles of 30 to $60 \mathrm{~nm}$ with eccentric cores and of unknown origin (Furness and Costa, 1980) were not identified unequivocally in our preparations. Similarly, although quite often seen in intact material (Furness and Costa, 1980), "type 3" terminals containing flattened, clear core vesicles were not encountered frequently in our varicosity preparations; these terminals are thought to be noradrenergic (Furness and Costa, 1980). Significantly, our myenteric varicosity preparations do contain noradrenaline (M. Alhumayyd and T. D. White, unpublished observation). The paucity of flattened vesicles observed in our preparations could be due to the different buffers employed during fixation, since vesicle shape does depend on the osmolarity of the buffer used in the fixative (Valdivia, 1971).

"Type 4" (P type) terminals containing numerous large granular vesicles, were quite common in our myenteric varicosity preparations. It has been suggested that these terminals contain peptides (Furness and Costa, 1980) or may be "purinergic" (Burnstock, 1979).

The "type 5" terminal of Furness and Costa (1980) is identified by performing a uranaffin reaction on the tissue (Richards and Da Prada, 1977). This technique was not performed on our preparations. "Type 6" terminals containing many small mitochondria and thought to be sensory in function were rare in our preparations. Varicosities containing glycogen ("type 7") and varicosities with dense cytoplasm ("type 8"), the latter possibly representing degenerating nerve endings (Furness and Costa, 1980), were observed only occasionally in our preparations.

Finally, in contrast to another fine structural study of varicosities prepared from guinea pig myenteric plexus (Jonakait et al., 1979), some of our nerve terminals contained neurotubules. Differences in the fixation procedures used could account for this difference. In any event, our relatively simple procedure for preparing and isolating myenteric varicosities yields preparations which are morphologically comparable, if not superior, to those preparations described previously (Jonakait et al., 1979; Dowe et al., 1980; Briggs and Cooper, 1981). Moreover, our preparations contain most of the types of nerve endings found in intact guinea pig myenteric plexus (Furness and Costa, 1980).

Release of ATP. Both crude and purified preparations of myenteric varicosities released ATP when they were depolarized with elevated extracellular $\mathrm{K}^{+}$or with the veratrum alkaloid, veratridine. It is most unlikely that release of ATP occurred from mitochondria contaminating the preparations, since purified mitochondrial preparations exhibited very little release. The $\mathrm{K}^{+}$-induced release of ATP from myenteric varicosities closely resembled, in onset, duration, monovalent cation specificity, and its requirement for extracellular $\mathrm{Ca}^{2+}$, the $\mathrm{K}^{+}$-induced release of ATP from rat brain synaptosomes reported previously (White, 1977, 1978).

Veratridine, which specifically depolarizes excitable 
a

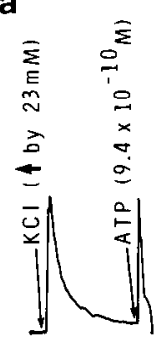

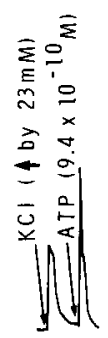
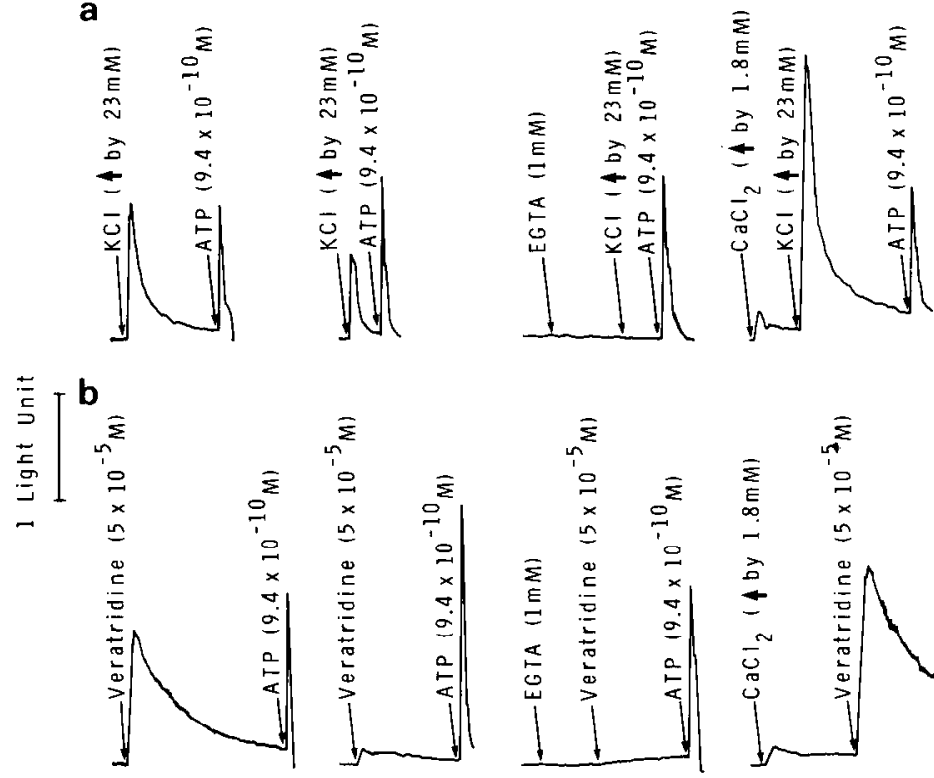

NORMAL

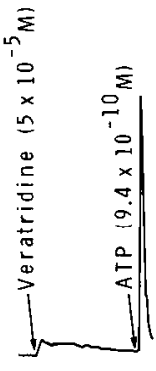

$C a^{2+}-F R E E$
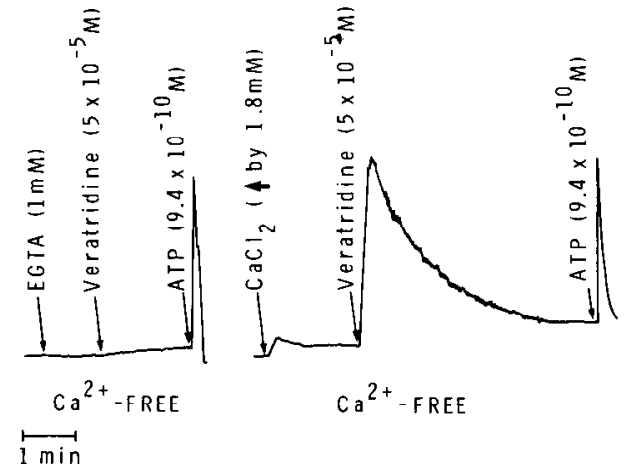

Figure 6. Effect of extracellular $\mathrm{Ca}^{2+}$ on depolarization-induced release of ATP from myenteric varicosities. Crude myenteric varicosities $\left(\mathbf{P}_{2}\right)$ were suspended in normal medium or in a medium lacking $\mathrm{CaCl}_{2}\left(\mathrm{Ca}^{2+}\right.$ free) and preincubated for 30 min. $a$, Release of ATP by $23 \mathrm{~mm} \mathrm{KCl}$ was determined for normal varicosities, for the $\mathrm{Ca}^{2+}$-free suspension, for the suspension to which 1 mim EGTA had been added to chelate residual $\mathrm{Ca}^{2+}$, and for the $\mathrm{Ca}^{2+}$-free suspension in which $\mathrm{CaCl}_{2}$ was restored to $1.8 \mathrm{mM}$. As an internal standard, ATP in $\mathrm{Ca}^{2+}$-free incubation medium was injected to give $9.4 \times 10^{-10} \mathrm{M}$ ATP in the cuvette. $b$, Release of ATP by $5 \times 10^{-5} \mathrm{M}$ veratridine was determined as in $a$ above. The results shown were observed in five experiments.

cells by opening the $\mathrm{Na}^{+}$channels in their cell membranes (Ulbricht, 1969), caused a rapid release of ATP from myenteric varicosities. In contrast to the release from rat brain synaptosomes (White, 1978), the veratridine-induced release of ATP from myenteric varicosities was shorter in duration and required extracellular $\mathrm{Ca}^{2+}$. This requirement for extracellular $\mathrm{Ca}^{2+}$ in the case of myenteric varicosities was not seen in the case of veratridine-induced release of ATP from guinea pig brain synaptosomes, where veratridine-induced release of ATP was increased rather than decreased in the absence of extracellular $\mathrm{Ca}^{2+}$. Clearly, the veratridine-induced release of ATP from myenteric varicosities differs fundamentally from release from brain synaptosomes and we are currently investigating the reasons for these differences.

Numerous studies have demonstrated that the depolarization-induced release of a number of putative neurotransmitters from synaptosomes requires extracellular $\mathrm{Ca}^{2+}$ (Blaustein et al., 1972; DeBelleroche and Bradford, 1972). Recently, Briggs and Cooper (1981) have demonstrated a $\mathrm{Ca}^{2+}$-dependent release of $\left[{ }^{3} \mathrm{H}\right]$ acetylcholine from myenteric varicosities depolarized with $\mathrm{K}^{+}$or veratridine. The depolarization-induced, $\mathrm{Ca}^{2+}$-dependent release of ATP from myenteric varicosities described above closely resembles a typical neurotransmitter release process and is consistent with a possible neurotransmitter function for ATP in this tissue. On the other hand, there is evidence that ATP may be stored with acetylcholine and noradrenaline within their respective synaptic vesicles (Dowdall et al., 1974; Geffen and Livett, 1971). In light of the morphological evidence that both adrenergic and cholinergic as well as possible "purinergic" varicosities are present in our myenteric preparations, it seems possible that ATP could be released during the expulsion of these other neurotransmitters. If this is so, then the released ATP might function as a co-transmitter or modulator rather than as a primary neurotransmitter.

In any case, it seems unlikely that an energetically valuable molecule such as ATP would be released merely as an accident of exocytosis with no functional significance. Certainly low concentrations of exogenously applied ATP cause relaxations in intestinal smooth muscle preparations (Burnstock et al., 1970). Experiments are in progress to determine whether ATP is co-released with some specific neurotransmitter or is possibly released as a "purinergic" neurotransmitter in its own right. 

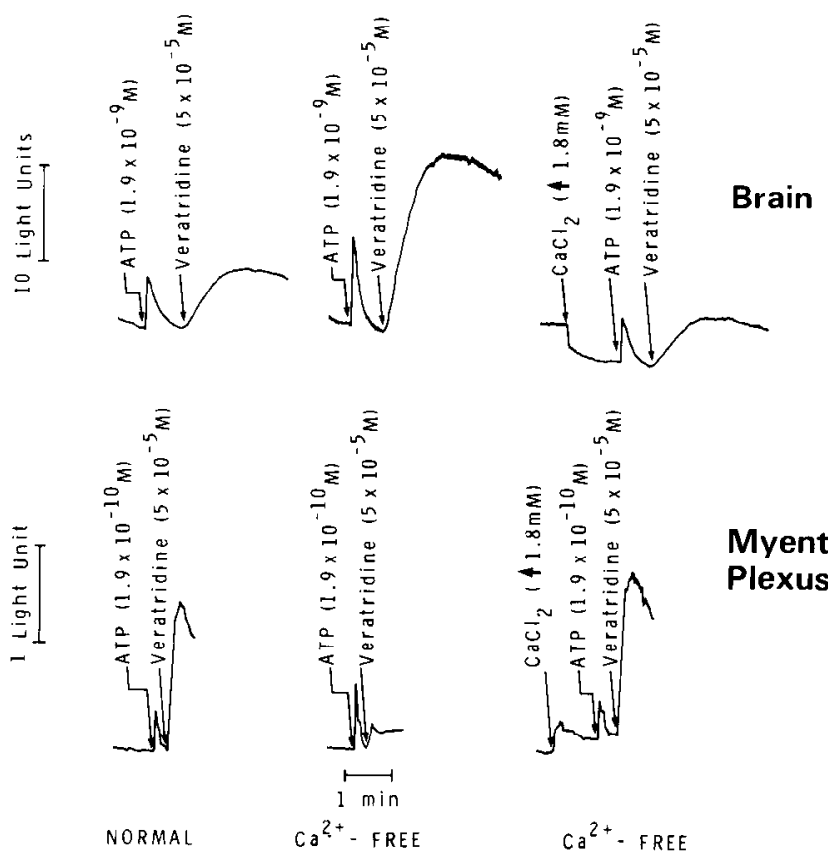

NORMAL

$$
C a^{2+}-F R E E
$$

Myenteric Plexus

Figure $7 . \mathrm{Ca}^{2+}$ requirements for the veratridine-induced release of ATP from brain and myenteric terminals. Crude synaptosomal preparations $\left(\mathrm{P}_{2}\right)$ were isolated from the brain and myenteric plexus of guinea pigs as described under "Materials and Methods" and were suspended in normal medium or in a medium lacking $\mathrm{CaCl}_{2}\left(\mathrm{Ca}^{2+}\right.$ free $)$ and preincubated for $30 \mathrm{~min}$. The release of ATP by $5 \times 10^{-5} \mathrm{M}$ veratridine was determined for normal synaptosomes, for $\mathrm{Ca}^{2+}$-free synaptosomes, and for $\mathrm{Ca}^{2+}$-free suspensions to which $\mathrm{CaCl}_{2}$ was restored to $1.8 \mathrm{mM}$. As an internal standard, ATP in $\mathrm{Ca}^{2+}$-free medium was injected to give $1.9 \times 10^{-9} \mathrm{M}$ ATP in the case of brain synaptosomes or $1.9 \times 10^{-10} \mathrm{M}$ ATP in the case of myenteric varicosities. The results shown were observed in three experiments.

\section{References}

Blaustein, M. P., E. M. Johnson, and P. Needleman (1972) Calcium-dependent norepinephrine release from presynaptic nerve endings in vitro. Proc. Natl. Acad. Sci. U. S. A. 69: 2237-2240.

Briggs, C. A., and J. R. Cooper (1981) A synaptosomal preparation from the guinea pig ileum myenteric plexus. J. Neurochem. 36: 1097-1108.

Burnstock, G. (1979) Past and current evidence for the purinergic nerve hypothesis. In Physiological and Regulatory Functions of Adenosine and Adenine Nucleotides, H. P. Baer and G. I. Drummond, eds., pp. 3-32, Raven Press, New York.

Burnstock, G., G. Campbell, D. G. Satchell, and A. Smythe (1970) Evidence that adenosine triphosphate or a related nucleotide is the transmitter released by non-adrenergic inhibitory nerves in the gut. Br. J. Pharmacol. 40: 668-688.

Burnstock, G., T. Cocks, L. Kasakov, and H. K. Wong (1978) Direct evidence for A'TP release from non-adrenergic, noncholinergic ("purinergic") nerves in the guinea-pig Taenia coli and bladder. Eur. J. Pharmacol. 49: 145-149.

DeBelleroche, J. S., and H. F. Bradford (1972) The stimulusinduced release of acetylcholine from synaptosome beds and its calcium dependence. J. Neurochem. 19: 1817-1819.
Dowdall, M. J., A. F. Boyne, and V. P. Whittaker (1974) Adenosine triphosphate-a constituent of cholinergic synaptic vesicles. Biochem. J. 140: 1-12.

Dowe, G. H. C., H. Kilbinger, and V. P. Whittaker (1980) Isolation of cholinergic synaptic vesicles from the myenteric plexus of guinea-pig small intestine. J. Neurochem. 35: 9931003.

Feng, T. P., and Y. M. Liu (1949) The concentration-effect relationship in the depolarization of amphibian nerve by potassium and other agents. J. Cell. Comp. Physiol. 34: 3342.

Furness, J. B., and M. Costa (1980) Types of nerves in the enteric nervous system. Neuroscience 5: 1-20.

Geffen, L. B., and B. C. Livett (1971) Synaptic vesicles in sympathetic neurones. Physiol. Rev. 51: 98-157.

Hartree, E. F. (1972) Determination of protein: A modification of the Lowry method that gives a linear photometric response. Anal. Biochem. 48: 422-427.

Johnson, M. K., and V. P. Whittaker (1963) Lactate dehydrogenase as a cytoplasmic marker in brain. Biochem. J. 88: 404409.

Jonakait, G. M., A. R. Gintzler, and M. D. Gershon (1979) Isolation of axonal varicosities (autonomic synaptosomes) from the enteric nervous system. J. Neurochem. 32: 13871400 .

Narahashi, T., J. W. Moore, and W. R. Scott (1964) Tetrodotoxin blockage of sodium conductance increase on lobster giant axons. J. Gen. Physiol. 47: 965-974.

Paton, W. D. M., and M. A. Zar (1968) The origin of acetylcholine released from guinea-pig intestine and longitudinal muscle strips. J. Physiol. (Lond.) 194: 13-33.

Potter, P., and T. D. White (1980) Release of adenosine 5'triphosphate from synaptosomes from different regions of rat brain. Neuroscience 5: 1351-1356.

Reynolds, E. S. (1963) The use of lead citrate at high $\mathrm{pH}$ as an electron-opaque stain in electron microscopy. J. Cell Biol. 17: 208-212.

Richards, J. G., and M. Da Prada (1977) Uranaffin reaction: A new cytochemical technique for the localization of adenine nucleotides in organelles storing biogenic amines. J. Histochem. Cytochem. 25: 1322-1336.

Rutherford, A., and G. Burnstock (1978) Neuronal and nonneuronal components in the overflow of labelled adenyl compounds from guinea-pig Taenia coli. Eur. J. Pharmacol. 48: 195-202.

Satchell, D. G., and G. Burnstock (1971) Quantitative studies of the release of purine compounds following stimulation of non-adrenergic inhibitory nerves in the stomach. Biochem. Pharmacol. 20: 1694-1697.

Su, C., J. A. Bevan, and G. Burnstock (1971) [ $\left.{ }^{3} \mathrm{H}\right]$ Adenosine triphosphate: Release during stimulation of enteric nerves. Science 173: 337-339.

Ulbricht, W. (1969) The effect of veratridine on excitable membranes of nerve and muscle. Ergeb. Physiol. Biol. Chem. Exp. Pharmacol. 61: 19-61.

Valdivia, O. (1971) Methods of fixation and the morphology of synaptic vesicles. J. Comp. Neurol. 142: 257-274.

White, T. D. (1977) Direct detection of depolarisation-induced release of ATP from a synaptosomal preparation. Nature 267: 67-68.

White, T. D. (1978) Release of ATP from a synaptosomal preparation by elevated extracellular $\mathrm{K}^{+}$and by veratridine. J. Neurochem. 30: 329-336.

White, T. D., and D. Webb (1980a) Depolarization-induced release of ATP from "autonomic synaptosomes" prepared from guinea pig myenteric plexus. Proc. Can. Fed. Biol. Soc. 23: 83 . 
White, T. D., and D. Webb (1980b) Release of ATP from "autonomic synaptosomes" prepared from the myenteric plexus of guinea-pig ileum. Soc. Neurosci. Abstr. 6: 625.

White, T., P. Potter, and S. Wonnacott (1980) Depolarisationinduced release of ATP from cortical synaptosomes is not associated with acetylcholine release. J. Neurochem. 34: 1109-1112.
Whittaker, V. P., and L. A. Barker (1972) The subcellular fractionation of brain tissue with special reference to the preparation of synaptosomes and their component organelles. In Methods of Neurochemistry, R. Freid, ed., Vol. 2, pp. 152, Dekker, New York.

Wroblewski, F., and J. S. LaDue (1955) Lactate dehydrogenase activity in blood. Proc. Soc. Exp. Biol. Med. 90: 210-213. 